\title{
educação

\section{Evidências de validade para a Escala de Vitimização entre Alunos (EVA)}

\author{
Ana Carina Stelko-Pereira ${ }^{1}$
}

Universidade Federal do Paraná, Brasil

\author{
Lúcia Cavalcanti de Albuquerque Williams ${ }^{\text {II }}$ \\ Universidade Federal de São Carlos, Brasil \\ Rodolfo Augusto Matteo Ambie ${ }^{\text {III }}$ \& Pedro \\ Afonso Cortez IV \\ Universidade São Francisco, Brasil
}

\begin{abstract}
Este estudo avalia a Escala de Vitimização entre Alunos (EVA) quanto à validade da sua estrutura interna, consistência interna, e na relação com outra variável (stress). Para tal, foram analisadas respostas à EVA de 1.484 alunos de escolas públicas do 6.0 ao 9.o ano do Ensino Básico provenientes de seis cidades de quatro estados do Brasil. Notou-se que a escala é formada por dois fatores: vitimização presencial e vitimização virtual, os quais conjuntamente explicam cerca de $50 \%$ da variância, obtendo um índice de consistência interna de 0,84 e 0,77 para os fatores. Adicionalmente, na cidade de Fortaleza $(\mathrm{N}=100)$ aplicou-se a EVA duas vezes num intervalo de 30 dias, obtendo-se um índice de precisão para o valor global de 0,64, e aplicou-se a Escala de Stress Infantil (ESI), tendose percebido correlações positivas significativas superiores a 0,65 entre os valores da EVA e da ESI. Assim, a EVA é uma alternativa promissora.
\end{abstract}

Palavras-chave: Bullying; Intimidação escolar; Psicometria

\section{N T RO D U Ç Ã O}

A violência na escola entre alunos é um grave problema, tendo sido apontado como frequente em diversas regiões do Brasil (Abramovay, Castro, Silva, \& Cerqueira, 2016; Instituto Brasileiro de Geografia e Estatística [IBGE], 2016). Esta pode envolver um episódio pontual, como a troca de socos devido à discordância num jogo de futebol, ou ainda vários episódios em que ocorre a perseguição de um aluno por outro(s) aluno(s) em situação desigual de poder, sendo o último exemplo denominado bullying (Olweus, 2013).

Os atos pontuais ou crónicos de violência entre pares podem ocorrer de modo presencial, quando a vítima identifica mais facilmente o autor da agressão, ou de modo virtual, como no caso do cyberbullying (StelkoPereira, Albuquerque, \& Williams, 2015). Em relação à vitimização, os alunos podem ser exclusivamente vítimas ou vítimas-autores. As vítimas exclusivas podem ser fisicamente mais fracas e/ou impopulares - com poucos amigos na escola e poucas habilidades para lidar com situações conflituosas (Khoury- 
Kassabri, Benbenishty, Astor, \& Zeira, 2004). Em contraste, as vítimas-autoras apresentam dificuldade em controlar a impulsividade e em avaliar contextos sociais, acabando por agredir colegas que, subsequentemente, terão condições de retaliar a agressão sofrida e/ou ser violentos em frente de adultos (Schwartz, Proctor, \& Chien, 2001). Cabe saber que o papel da vítima é relativamente estável, conforme meta-análise de 77 estudos longitudinais (Pouwels, Souren, Lansu, \& Cillessen, 2016).

A violência presencial ou virtual entre alunos pode acarretar diversos impactos negativos à saúde dos envolvidos. Vítimas de bullying têm maior probabilidade de desenvolver transtornos depressivos e se suicidar (Forlim, Stelko-Pereira, \& Williams, 2014; Holt et al., 2015), apresentar sintomas de stress (Sousa \& Stelko-Pereira, 2016) e baixo engajamento escolar (Valle, Stelko-Pereira, Sá, \& Williams, 2015), sendo que o impacto na vítima pode afetar a vida adulta (Wolke \& Lereya, 2015).

Devido à importância do fenómeno, na Lei Brasileira de n. ${ }^{\circ} 13.185$, de 6 de novembro de 2015, institui-se, no seu artigo $5 .^{\circ}$, que é dever dos estabelecimentos de ensino, clubes e associações recreativas diagnosticar situações de bullying e, no seu $6 .^{\circ}$ artigo, indica-se que deverão ser elaborados relatórios bimestrais municipais e estatais da ocorrência destas situações. Assim, é de extrema relevância que haja instrumentos adequados para a realização da aferição do fenómeno no Brasil.

Desde o desenvolvimento da área de investigação sobre violência entre alunos e bullying, que ocorreu sobretudo após a década de 80 , as pesquisas de levantamento sobre a frequência da ocorrência, a forma da ocorrência, os fatores de risco e de proteção associados e, ainda, as pesquisas sobre a efetividade das intervenções têm-se baseado em dados coletados através de questionários. Contudo, diversos autores (Felix, Sharkey,
Green, Furlong, \& Tanigawa, 2011; Hamburger, Basile, \& Vivolo, 2011) têm apontado a carência de estudos de validade e precisão dos instrumentos.

Os poucos estudos de avaliação de instrumento de bullying disponíveis na literatura tendem a investigar a consistência interna das dimensões violência física, verbal, social/relacional (Hulsey, 2005; Kyriakides, Kaloyirou, \& Lindsay, 2006). No entanto, também há estudos em que outras dimensões foram investigadas. Por exemplo, Mynard e Joseph (2000) notaram no instrumento Multidimensional Peer Victimization Scale quatro fatores: vitimização física, social, verbal e dano à propriedade, todos com $\alpha>0,72$, e Cheng, Chen, Liu, e Chen (2011) investigaram o que é ser autor, vítima e testemunha de bullying físico, verbal, relacional e cyberbullying.

Já quanto a validade convergente para os instrumentos de avaliação de situações de violência entre alunos, pode investigar-se a convergência entre instrumentos de avaliação de vitimização entre colegas através de instrumentos que avaliam ansiedade, stress e depressão, pois há estudos que demonstraram relações entre tais constructos (por exemplo: Forlim et al., 2014; Turner, Reynolds, Lee, Subasic, \& Bromhead, 2014; Yen, Lin, Liu, Hu, \& Cheng, 2014).

Em relação à realidade brasileira, o panorama de instrumentos disponíveis para avaliação de violência entre alunos é semelhante à realidade internacional quanto à escassez de estudos psicométricos (Alckmin-Carvalho, Izbicki, Fernandes, \& Melo, 2014). Entre os poucos instrumentos sobre vitimização entre alunos investigados no Brasil, destaca-se o de Cunha (2009), denominado "Escala de Agressão e Vitimização entre Pares - EVAP", o qual contém 18 itens que questionam sobre os comportamentos agressivos referentes aos últimos 6 meses, em escala Likert de 5 
pontos. Esta escala apresenta quatro fatores (agressão direta, agressão relacional, agressão física indireta e vitimização), que explicam $54,62 \%$ da variância. Contudo, pode afirmarse que a escala precisa de aprimoramentos, visto que o fator "Agressão física indireta" apenas apresenta um item (referente a roubar ou mexer nas coisas do colega) e os fatores "Agressão relacional" e "Agressão direta" não estão plenamente coerentes do ponto de vista teórico. Por exemplo, os itens "colocar apelidos depreciativos" e "fazer comentários depreciativos" encontram-se no fator "Agressão relacional", mas são semelhantes ao item "gozar/chatear", que se situa no fator "Agressão direta". Apesar dessas discrepâncias, foram obtidos índices de consistência interna satisfatórios $(\alpha>0,70)$ em dois fatores.

Outro instrumento é a Escala de Avaliação de Bullying Escolar (EAB), elaborado por Silva (2014), cujo público-alvo são indivíduos dos 6 aos 20 anos. Esta escala tem 47 itens, cujas alternativas de resposta são: "Nunca" "Às vezes" e "Sempre", com dois fatores: autores e vítimas, os quais explicam cerca de $48,46 \%$ da variância dos dados. A escala apresentou validade convergente com a Escala de Autoconceito Infanto-Juvenil e índices adequados de fidelidade com relação ao coeficiente de Alfa de Cronbach (vítimas $=0,88$ e autores $=0,84$ ). Entretanto, o instrumento não contempla estudos de estabilidade ao longo do tempo.

Um terceiro instrumento estrangeiro foi adaptado para o Brasil por Soares, Gouveia, Gouveia, Fonsêca, e Pimentel (2015) - a Escala Califórnia de Vitimização do Bullying (ECVB) -, a qual consta de sete itens que questionam se o indivíduo sofreu diversas ações de bullying numa escala de 5 pontos para, de seguida, questionar se estas ações foram intencionais por parte do autor, se causaram mal-estar e se o autor apresentava maior poder do que a vítima. Em análise fatorial com dados de estudantes da faixa etária dos 11 aos 16 anos residentes em João Pessoa, constatou-se ser a escala unidimensional, explicando $40,3 \%$ da variância, com uma consistência interna de 0,72 e confirmação da dimensão por análise restrita. Porém, no estudo de Soares et al. (2015) apontou-se a necessidade de estudos de evidências de validade da escala com relação a outras variáveis, da estabilidade das respostas ao longo do tempo e com uma amostra de participantes mais diversa.

Adicionalmente, um quarto instrumento brasileiro denomina-se Escala de Violência Escolar (EVE) (Stelko-Pereira \& Williams, 2016), o qual possui versões para diversos respondentes (alunos, professores e diretores) e que originalmente foi denominado de Questionário de Investigação de Prevalência de Violência Escolar (QIPVE) (Stelko-Pereira, Williams, \& Freitas, 2010). A versão para alunos tem sido investigada e aprimorada, sendo que a quantidade de itens e os aspectos da violência escolar em análise aumentaram, de modo que a versão foi desmembrada em três escalas: Escala de Vitimização entre Alunos (EVA); Escala de Vitimização a Alunos por Funcionários (EVAF); e Escala de Autoria de Violência de Alunos (EAVA). Diante tais modificações, considera-se necessário avaliar as características psicométricas de cada uma destas escalas separadamente, com o intuito de assegurar a qualidade das inferências realizadas por meio delas.

Para prestar contribuições ao tema, o objetivo da corrente investigação foi analisar evidências de validade baseadas na estrutura interna para a Escala de Vitimização entre Alunos (EVA), o nível de consistência interna dos fatores da medida, bem como verificar a relação desta com uma variável externa (stress). 


\section{MÉTODO}

\subsection{PARTICIPANTES}

Participaram no estudo 1.484 estudantes, sendo $52 \%$ do sexo feminino e $48 \%$ do sexo masculino, com média de idade igual a 13 $(\mathrm{DP}=1,37)$ anos, variando entre 10 e 17 anos. Desses alunos, $27,9 \%$ encontravam-se no $6 .^{\circ}$ ano, $28,8 \%$ no $7 .^{\circ}$ ano, $28,6 \%$ no $8 .^{\circ}$ ano e $15,1 \%$ no $9 .^{\circ}$ ano do Ensino Básico. Os estudantes residiam em seis diferentes cidades, estudando em escolas públicas, sendo que 57,4\% eram de Curitiba (PR), 16,7\% de Sumaré (SP), 10,8\% de São Paulo (SP), 9,6\% de Fortaleza (CE) e $5,4 \%$ de São Carlos (SP).

\subsection{INSTRUMENTOS}

Escala de Vitimização entre Alunos - EVA: instrumento de autorrelato com 18 itens e escala de resposta do tipo Likert de 5 pontos ( 1 = "nenhuma vez" e 5 = "sete vezes ou mais"). Esta escala procura avaliar a ocorrência de situações em que os alunos são alvo de violência por outros alunos do 5.o ao 9.o ano do Ensino Básico (incluindo ou não ter sido alvo de bullying) nos últimos 6 meses. $\mathrm{O}$ instrumento pode ser respondido individualmente ou coletivamente, em cerca de 20 minutos. O fator vitimização presencial é composto por 11 itens que avaliam a ocorrência de violência na realidade concreta do escolar e o fator vitimização virtual possui sete itens com situações de violência por meio de domínios digitais.

Escala de Stress Infantil - ESI (Lipp \& Lucarelli, 2003): instrumento de autorrelato com 35 itens e escala de resposta do tipo Likert de 4 pontos variando quanto à frequência de ocorrência do conteúdo stressante. Esta escala tem como objetivo avaliar a ocorrência de stress em crianças. Alguns exemplos de itens são "Sinto aflição por dentro" ou "Quando fico nervoso, gaguejo". No presente estudo, optouse por analisar o stress infantil pelo valor total gerado para avaliação do fenómeno como unidimensional. A análise de consistência interna da ESI aponta para um valor de Alfa de Cronbach igual a 0,75 . Tal instrumento foi aplicado no presente estudo apenas na amostra da cidade de Fortaleza $(\mathrm{N}=100)$.

\subsection{PROCEDIMIENTO}

Recolha de dados: A primeira autora deste artigo realizou a recolha de dados em Fortaleza. Já nas outras cidades, investigadores parceiros, que pretendiam empregar $o$ instrumento para pesquisas de mestrado, com diferentes objetivos, utilizaram-no, tendo cedido os dados para esta análise. A recolha de dados, nas várias cidades, foi realizada através do preenchimento dos questionários em horário letivo e de forma coletiva, em turmas de escolas selecionadas por conveniência pelos investigadores.

Análise de dados: $O$ método de retenção fatorial empregado foi a análise paralela. $\mathrm{Na}$ análise fatorial foi implementado um modelo saturado para identificar a estrutura fatorial da medida. Estas análises foram realizadas por meio do software Factor 10.8.04 com a utilização de correlações policóricas e estimador robusto a dados ordinais (ULS). A consistência interna foi avaliada através do Alfa de Cronbach. As correlações de Spearman foram aplicadas para avaliar o nível de correlação entre os valores por dimensões e o total referente à EVA nos dois momentos (fidedignidade teste-reteste), tendo-se usado apenas as respostas dos alunos de Fortaleza (único local onde se coletaram dados para avaliar estabilidade temporal). $\mathrm{O}$ modelo fatorial restrito também foi gerado com o uso de correlações policóricas e estimador robusto a dados ordinais (ULS). Essas análises 
foram geradas no software JASP versão 0.8.4.0.

\subsection{CONSIDERAÇões ÉTICAS}

Este estudo foi aprovado pelo Comitê de Ética em Pesquisa com Seres Humanos da Universidade Estadual do Ceará (CAAE 25377913.6.0000,5534), permitindo a recolha de dados em Fortaleza e a análise de dados de outros estudos, os quais também obtiveram aprovação para seus objetivos específicos: Universidade Tuiuti do Paraná (CAAE 12501513.9.00000103), Universidade Federal de São Carlos (CAAE 12487813.2.0000,5504) e Universidade de São Paulo (CAAE 0474191330000,5561). Os participantes assinaram o Termo de Consentimento e os seus responsáveis legais um Termo de Consentimento Livre Esclarecido, autorizando-os a participar.

TABELA 1

Estrutura Fatorial Saturada Identificada para a EVA $(N=1.484)$

\begin{tabular}{llcc}
\hline Item & & \multicolumn{1}{c}{ Descrição } & F1 \\
\hline i1 & Ter materiais destruídos propositadamente & $\mathbf{0 , 5 1}$ & 0,04 \\
i2 & Ser roubado ou furtado & $\mathbf{0 , 4 3}$ & 0,08 \\
i3 & Ser alvo de rumores & $\mathbf{0 , 4 9}$ & 0,10 \\
i4 & Ser excluído propositadamente & $\mathbf{0 , 3 7}$ & 0,27 \\
i5 & Ser gozado propositadamente & $\mathbf{0 , 6 8}$ & $-0,07$ \\
i6 & Receber ameaças físicas & $\mathbf{0 , 6 0}$ & 0,18 \\
i7 & Receber ameaças psicológicas & $\mathbf{0 , 6 5}$ & 0,11 \\
i8 & Receber tapas & $\mathbf{0 , 7 3}$ & $-0,03$ \\
i9 & Receber socos & $\mathbf{0 , 7 7}$ & $-0,05$ \\
i10 & Ser chutado & $\mathbf{0 , 7 4}$ & 0,04 \\
i11 & Alguém fazer tropeçar & $\mathbf{0 , 7 4}$ & $-0,21$ \\
i12 & Alguém lhe enviar mensagens xingando & $-0,13$ & $\mathbf{0 , 7 2}$ \\
i13 & Alguém lhe enviar mensagens ameaçando fisicamente & 0,19 & $\mathbf{0 , 5 1}$ \\
i14 & Alguém lhe enviar mensagens ameaçando de ridicularizar & $-0,06$ & $\mathbf{0 , 8 1}$ \\
i15 & Alguém publicar mensagens para lhe ofender & 0,27 & $\mathbf{0 , 4 3}$ \\
i16 & Alguém publicar vídeo o ofendendo & $-0,17$ & $\mathbf{0 , 9 3}$ \\
i17 & Alguém publicar vídeo em que o indivíduo foi ridicularizado & 0,16 & $\mathbf{0 , 5 6}$ \\
i18 & Alguém fingir ser a pessoa enviando mensagens e vídeos para prejudicar & 0,18 & $\mathbf{0 , 5 2}$ \\
& Variância explicada por fator (\%) & $39,3 \%$ & $10,8 \%$ \\
& alfa de cronbach & 0,84 & 0,77 \\
& IC [0,95\%] & {$[0,83 \sim 0,86]$} & {$[0,75 \sim 0,79]$} \\
\hline
\end{tabular}

Nota. F1 = Vitimização presencial; F2 = Vitimização virtual; IC = Intervalo de confiança para Alfa de Cronbach

\section{RESULTADOS}

Em relação ao objetivo do estudo de avaliar a EVA quanto à validade da sua estrutura interna, inicialmente fez-se a análise de todos os itens da Escala de Vitimização Presencial entre Alunos conjuntamente, tendo sido constatado que os dados obedeciam aos preceitos requeridos para a realização de uma análise fatorial

(KMO $=0,73)$. Em seguida, verificou-se por análise paralela a indicação de retenção de dois fatores (Auto valor simulado = 1,15; Auto valor empírico $=1,94)$, tal como hipotetizado teoricamente. Com isto, foi implementada a análise fatorial com especificação de modelo saturado, extração de dois fatores e rotação varimax. A estrutura fatorial identificada é apresentada na Tabela 1, a seguir.

Conforme a Tabela 1, a EVA apresentou um total de 18 itens, sendo que 11 desses investigam a vitimização presencial entre alunos e sete investigam a vitimização virtual. Nenhum item saturou em mais de um fator, ou seja, nenhum 
item teve carga fatorial superior a 0,40 em mais de um fator. Em relação à análise da precisão da EVA, o fator relativo à "vitimização presencial" (Alfa de Cronbach $=0,84$ ) teve maior consistência interna do que o fator "vitimização virtual" (Alfa de Cronbach=0,77).

A etapa seguinte foi a especificação de uma nova análise fatorial para verificar se a estrutura fatorial identificada possuía um nível de ajuste adequado ao restringir o cruzamento de cargas por meio de um modelo fatorial restrito. $\mathrm{O}$ primeiro fator de "vitimização presencial" foi especificado pelos itens il até i11 e o segundo fator abrangeu os itens i12 até i18, conforme verificado no modelo saturado. Adicionalmente, foi proposta correlação entre os fatores de primeira ordem da medida para verificar a estabilidade fatorial do instrumento ao integrar informação partilhada entre ambos. $\mathrm{O}$ modelo fatorial restrito é disposto na Figura 1.

O modelo fatorial restrito apresentou razoável robustez quanto aos índices de ajuste $\left(\mathrm{X}^{2} / \mathrm{gl}\right.$ $=337,93, \mathrm{df}=134, \mathrm{p}<0,01 ; \mathrm{CFI}=0,983$; TLI = 0,980; RMSEA $=0,032$ ). As cargas fatoriais para o primeiro fator de "vitimização presencial" oscilaram entre níveis adequados $(\lambda=0,44 \sim$ 0,67). O mesmo aconteceu com o segundo fator de "vitmização virtual", cujas cargas fatoriais abrangeram índices razoáveis $(\lambda=0,41 \sim 0,65)$. O nível de correlação entre os fatores também se mostrou adequado $(r=0,61 ; p<0,01)$, tendo em vista que as informações avaliadas entre ambos abrangem conteúdos semelhantes em domínios distintos.

$\mathrm{Na}$ averiguação da validade da EVA na sua relação com outra variável, as análises da EVA e da ESI indicaram correlações positivas moderadas, conforme a seguinte Tabela 2. A maior correlação entre as escalas foi quanto aos valores totais de $\operatorname{ambas}(r=0,70, p<0,01)$. A menor correlação foi entre o valor de "vitimização virtual" e as reações de stress infantil do tipo físico. A análise de

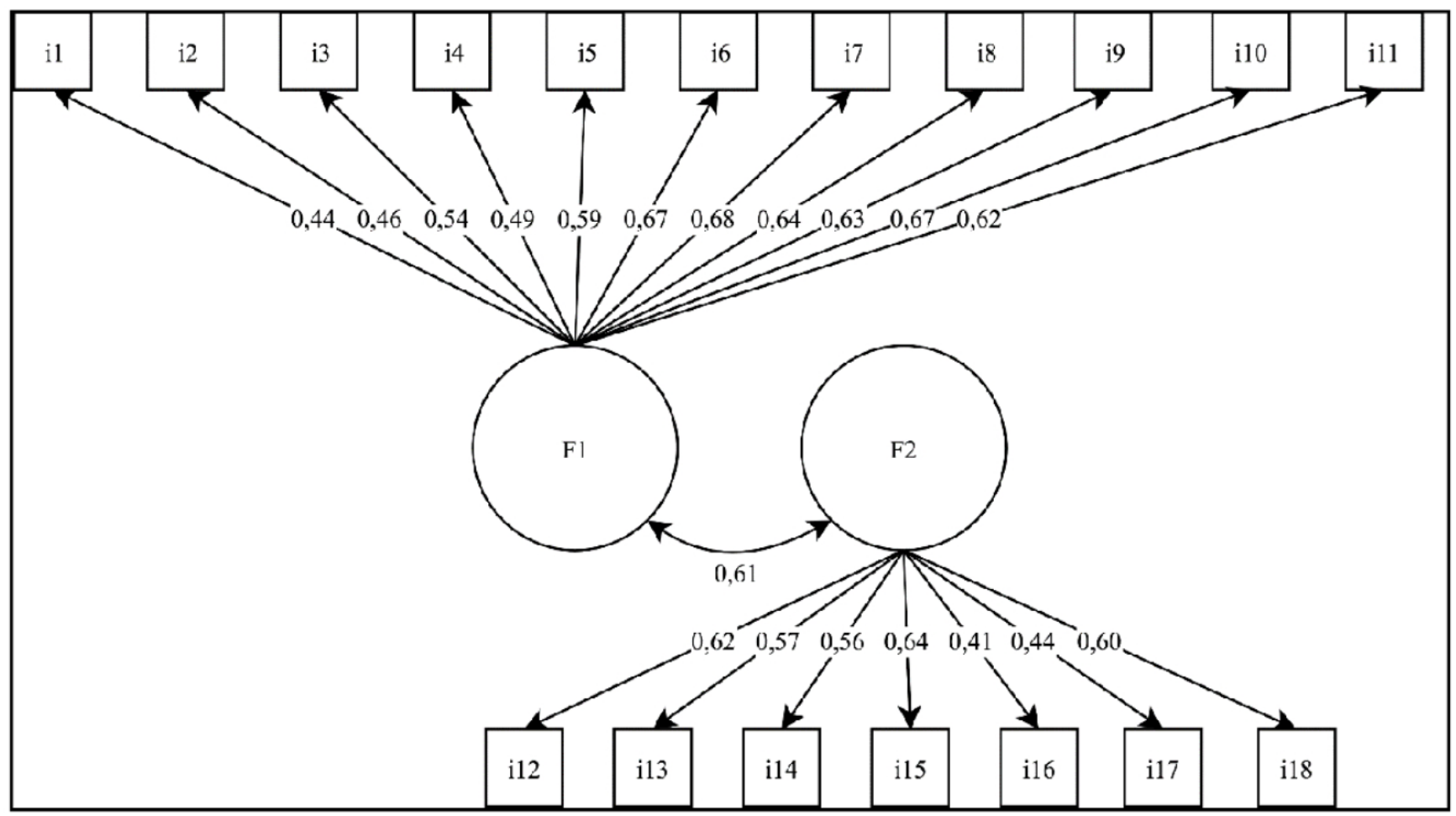

FIGURA 1. Estrutura fatorial restrita para a EVA $(\mathrm{N}=1484)$ 
TA B EL A 2

Correlações de Spearman entre a escala de vitimização entre pares e a escala de estresse infantil

\begin{tabular}{lllc}
\hline & \multicolumn{2}{c}{ Vitimização entre Alunos (N=100) } \\
& Presencial & Virtual & Total \\
\cline { 2 - 4 } $\begin{array}{c}\text { Escala de Estresse Infantil - Reações } \\
\text { Psicológicas } \\
\text { Com componentes depressivos }\end{array}$ & & & \\
Sem componentes depressivos & $0,60^{* *}$ & $0,63 * *$ & $0,65^{* *}$ \\
Psicofisiológicas & $0,56^{* *}$ & $0,60^{* *}$ & $0,68^{* *}$ \\
Físicas & $0,50^{* *}$ & $0,57 * *$ & $0,55^{* *}$ \\
Total & $0,57 * *$ & $0,46 * *$ & $0,50 * *$ \\
\hline
\end{tabular}

Nota. ${ }^{* *}$ Correlações significativas ao nível de 0,01 .

precisão por meio do teste-reteste num intervalo de 30 dias (para os participantes de Fortaleza) indicou que houve correlações de Spearman positivas significativas entre as aplicações de teste-reteste, seja em relação à vitimização presencial $(r=0,66, p<0,01)$, vitimização virtual $(\mathrm{r}=0,54, \mathrm{p}<0,01)$ ou vitimização total $(\mathrm{r}=0,64$, $\mathrm{p}<0,01)$.

\section{Is C U S S Ã O}

A Escala de Vitimização entre Alunos (EVA), componente da Escala de Violência Escolar (EVE), apresentou dois fatores teoricamente adequados (vitimização presencial e virtual) e com evidências empíricas satisfatórias, uma vez que se trata de fenómenos distintos, embora relacionados. Salienta-se como principal avanço proposto por esta investigação a identificação do fator "vitimização virtual", devido ao facto de atualmente ser comum o fácil acesso aos meios de comunicação pelos alunos, o que implica incluir a violência virtual do fenómeno da vitimização entre alunos (Donnerstein, 2012).
Os itens apresentaram coerência em relação às dimensões e explicaram cerca de $50 \%$ da variância, não tendo saturado em mais de um fator, de modo que se notou, assim, boa evidência de validade para a estrutura interna da medida. $\mathrm{O}$ instrumento abarcou questões que investigam situações de violência direta e indireta, cuja consequência predominante é a violência física, patrimonial e psicológica, tanto no âmbito presencial quanto virtual. Assim, pode dizer-se que a EVA contemplou itens representativos do fenómeno.

É interessante notar que, na análise dos itens da EVA, o método de retenção fatorial indicou que não foi pertinente separar em dimensões diferentes a violência presencial - por exemplo, decompor numa dimensão de violência física, verbal, social e relacional, como foi feito nas escalas investigadas por Hulsey (2005) e Kyriakides et al. (2006), ou decompor numa dimensão de violência física, verbal, social e dano à propriedade psicológica, como proposto por Mynard e Joseph (2000). Tão-pouco a EVA se mostrou um instrumento unidimensional como o investigado por Soares et al. (2015). 
Possivelmente, isto ocorreu porque na presente escala incluiu-se também questões de violência virtual, o que permitiu a ocorrência de dois fatores com maior ênfase nesses dois domínios.

Adicionalmente, na perspectiva teórica, é também possível argumentar que a divisão entre violência física e verbal é um tanto ou quanto arbitrária (Stelko-Pereira \& Williams, 2010), uma vez que atos como bater e agredir expressam significados e, portanto, podem ser considerados também uma forma de comunicação. Por outro lado, a violência verbal pode ter impacto no corpo dos alunos, através de sintomas psicossomáticos. Assim, frente às considerações teóricas estabelecidas e com o suporte empírico dos modelos analisados, parece plausível uma solução que abranja os dois fatores para a EVA.

Ressalta-se que, pelo facto do presente estudo ter analisado apenas a EVA da Escala de Violência Escolar, voltada exclusivamente para a vitimização, a sua comparação com os instrumentos brasileiros disponíveis de Cunha (2009) e de Silva (2014) foi dificultada, pois estes outros instrumentos envolvem análise fatorial das questões referentes tanto à vitimização quanto à autoria de violência. Apesar disso, considera-se esta uma decisão adequada, no sentido de que ao se analisar os itens da Escala de Violência Escolar separadamente, em diversas escalas, como a da EVA, consegue-se uma maior compreensão das características psicométricas de cada escala. Esse tipo de estratégia mostra-se adequado às directrizes teóricas da área, como observado na Escala Califórnia de Vitimização do Bullying que se restringiu à vitimização presencial (Soares at al., 2015), otimizando as propriedades avaliativas ao realizar um procedimento semelhante. Contudo, destaca-se a importância de se aplicar tanto escalas de vitimização quanto de autoria, de modo a que se possa perceber quando um aluno é simultaneamente vítima e autor.

Segundo critérios de Murphy e Davidshofer (2005), a EVA apresentou uma consistência interna adequada nos fatores vitimização presencial $(0,84)$ e virtual $(0,77)$. A validade da EVA baseada na relação com outras variáveis, como o stress (o qual foi medido pela ESI), mostrou-se adequada, uma vez que os valores totais de ambas obtiveram uma correlação de 0,70. Segundo Prieto e Muñiz (2000), correlações consideradas excelentes estariam acima de 0,6 , correlações boas variam entre 0,5 e 0,6 e as minimamente adequadas entre 0,4 e 0,5 . A menor correlação entre os valores das escalas foi de 0,46, entre vitimização virtual e reações físicas de stress. Assim, a existência de relação entre stress (seja nos seus componentes mais ansiosos ou mais depressivos) e sofrer violência por colegas na escola foi compatível com o descrito previamente pela literatura (Turner et al., 2014; Yen et al., 2014), indicando que o instrumento apresentou validade adequada na relação com outras variáveis.

Sobre a confiança teste-reteste, o instrumento apresentou-se favorável, com possibilidade de otimização. Segundo Prieto e Muñiz (2000), são necessárias correlações mínimas iguais ou superiores a 0,65. A EVA obteve correlações quanto aos valores de teste-reteste de vitimização presencial e total iguais a 0,66 e 0,65 , respectivamente; porém, a correlação entre teste-reteste com relação ao valor de vitimização virtual mostrou possibilidade de aprimoramento com índice igual a 0,55 .

Diante estes resultados, ficam algumas questões para investigações futuras: 1) Seria o constructo "violência entre pares", especialmente a violência entre alunos de modo virtual, pouco estável ao longo de um mês, ainda que o instrumento tenha questionado se nos últimos 6 meses os alunos sofreram situações de violência?; e 2) Sendo o fenómeno pouco estável, a aplicação entre teste-reteste deveria ocorrer num intervalo de tempo mais curto (como uma semana), para obter índices satisfatórios de estabilidade? Frente a tais dúvidas, para avaliar a efetividade 
de intervenções de cyberbullying, a EVA deve ser utilizada com cautela. Entretanto, mediante a escassez de instrumentos de medidas nacionais e o facto de nenhum dos instrumentos disponíveis ter avaliado a confiança teste-reteste, a EVA configura-se como uma alternativa, desde que a sua interpretação seja realizada com parcimónia.

Dentrealgumaslimitaçõesdoestudo,mencionase a impossibilidade de realizar o teste-reteste em toda a amostra, o que pode ser proposto em investigações futuras. Adicionalmente, as escolas participantes foram escolhidas por conveniência, o que contribui para o facto de que não são necessariamente representativas das regiões onde os dados foram coletados. Nesse sentido, ainda são necessárias outras investigações a respeito do instrumento, como estudos sobre validade de critério e validade divergente, bem como mais pesquisas quanto à estabilidade do instrumento. É relevante também que se elaborem normas para o instrumento, considerando a diversidade de regiões brasileiras, as particularidades entre escolas públicas e privadas, e variações de idade. Apesar destas limitações, conclui-se que a EVA é promissora, sendo mais uma opção de instrumento para o contexto brasileiro.

\section{REFERÊ NCIAS}

Abramovay, M., Castro, M. G., Silva, A. P., \& Cerqueira, L. (2016). Diagnóstico participativo da violência nas escolas: Falam os jovens. Rio de Janeiro, Brasil: FLACSOBrasil, OEI, MEC.

Alckmin-Carvalho, F., Izbicki, S., Fernandes, L. F. B., \& Melo, M. H. S. (2014). Estratégias e instrumentos para a identificação de bullying em estudos nacionais. Avaliação Psicológica, 13(3), 343-350. http://pepsic. bvsalud.org/scielo.php?script $=$ sci arttext\&pid=S1677-04712014000300006

Cheng, Y., Chen, L., Liu, K., \& Chen, Y. (2011). Development and psychometric evaluation of the school bullying scales: A Rasch measurement approach. Educational and Psychological Measurement, 71(1), 200-216. DOI:10.1177/0013164410387387

Cunha, J. M. (2009). Violência interpessoal em escolas no Brasil: Características e correlatos (Dissertação de mestrado). Recuperado de http://www.ppge.ufpr.br/teses/M09 cunha. pdf

Donnerstein, E. (2012). Internet bullying. Pediatric Clinics of North America, 59(3), 623-633. DOI:10.1016/j.pcl.2012.03.019

Felix, E. D., Sharkey, J. D., Green, J. G., Furlong, M. J., \& Tanigawa, D. (2011). Getting precise and pragmatic about the assessment of bullying: The development of the California Bullying Victimization Scale. Aggressive Behavior, 37(3), 234-247. DOI:10.1002/ ab.20389

Forlim, B. G., Stelko-Pereira, A. C., \& Williams, L. C. A. (2014). Relação entre bullying e sintomas depressivos em estudantes do ensino fundamental. Estudos de Psicologia, $31(3)$, 367-375. DOI:10.1590/0103166X2014000300005

Hamburger, M. E., Basile, K. C., \& Vivolo, A. M. (2011). Measuring bullying victimization, perpetration, and bystander experiences: $A$ compendium of assessment tools. Atlanta, G. A.: Centers for Disease Control and 
Prevention, National Center for Injury Prevention and Control.

Holt, M. K., Vivolo-Kantor, A. M., Polamin, J. R., Holland, K. M., DeGue, S., Matjasko, J., . . . Reid, G. (2015). Bullying and suicidal ideation and behaviors: A meta-analysis. Pediatrics, 135(2), e496-e509. DOI:10.1542/ peds.2014-1864

Hulsey, C. (2005). Examining the psychometric properties of self-report measures of bullying: Reliability of the peer relations questionnaire (Master's theses). Universidade do Kansas, Kansas, Estados Unidos

Instituto Brasileiro de Geografia e Estatística. (2016). Pesquisa nacional de saúde do escolar: 2015. Rio de Janeiro, Brasil: IBGE, Ministério do Planejamento, Desenvolvimento e Gestão (Cf: https://biblioteca.ibge.gov.br/ visualizacao/livros/liv97870.pdf)

Khoury-Kassabri, M., Benbenishty, R., Astor, R. A., \& Zeira, A. (2004). The contributions of community, family, and school variables to student victimization. American Journal of Community Psychology, 34(3), 187-204. DOI:10.1007/s10464-004-7414-4

Kyriakides, L., Kaloyirou, C., \& Lindsay, G. (2006). An analysis of the Revised Olweus Bully/Victim Questionnaire using the Rasch measurement model. British Journal of Educational Psychology, 76(4), 781-801. DOI:10.1348/000709905X53499

Congresso Nacional Brasileiro (2015). Lei n. ${ }^{\circ}$ 13.185, de 6 de novembro de 2015. Institui o Programa de Combate à Intimidação Sistemática (Bullying). Diário Oficial da União de 9 de novembro de 2015. http:// www.planalto.gov.br/ccivil 03/ ato20152018/2015/lei/l13185.htm

Lipp, M. E. N., \& Lucarelli, M. D. M. (2003). Escala de Stress Infantil. São Paulo, Brasil: Casa do Psicológo.

Murphy, K. R., \& Davidshofer, C. O. (2005). Psychological testing: Principles and applications. Upper Saddle River, NJ: Prentice-Hall, Inc.
Mynard, H., \& Joseph, S. (2000). Development of the Multidimensional Peer Victimization Scale. Aggressive Behavior, 26(2), 169-178. DOI:10.1002/(SICI)10982337(2000)26:23.0,CO;2-A

Olweus, D. (2013). School bullying: Development and some important challenges. Annual Review of Clinical Psychology, 9, 751-780. DOI:10.1146/ annurev-clinpsy-050212-185516

Pouwels, J. L., Souren, P. M., Lansu, T. A. M., \& Cillessen, A. H. N. (2016). Stability of peer victimization: A meta-analysis of longitudinal research. Developmental Review, 40, 1-24. DOI:10.1016/j.dr.2016.01.001

Prieto, G., \& Muñiz, J. (2000). Un modelo para evaluar la calidade de los tests utilizados en España. Papeles del Psicólogo, 77, 65-75. Recuperado de http://www.redalyc.org/ pdf/778/77807709.pdf

Schwartz, D., Proctor, L. J., \& Chien, D. H. (2001). The aggressive victim of bullying: Emotional and behavioral dysregulation as a pathway to victimization by peers. In J. Juvonen \& S. Graham (Orgs.), Peer harassment in school: The plight of the vulnerable and victimized (pp.147-174). New York, NY: The Guilford Press.

Silva, F. C. (2014). Escala de avaliação de bullying. São Paulo, Brasil: Vetor.

Soares, A. K. S., Gouveia, V. V., Gouveia, R. S. V., Fônseca, P. N., \& Pimentel, C. E. (2015). Escala Califórnia de Vitimização do Bullying (ECVB): Evidências de validade e consistência interna. Temas em Psicologia, 23(2), 481-491. DOI:10.9788/TP2015.2-18

Sousa, M. M. M., \& Stelko-Pereira, A. C. (2016). Relações entre violência escolar, gênero e estresse em pré-adolescentes. Revista Eletrônica de Educação, 10(1), 110127. DOI:10.14244/198271991304

Stelko-Pereira, A. C., Albuquerque, P. P., \& Williams, L. C. A. (2015). Como compreender e prevenir. In J. Abrusio (Org.), Educação digital (pp. 81-92). São 
Paulo, Brasil: Thomson Reuters.

Stelko-Pereira, A. C., \& Williams, L. C. A. (2010). Sobre o conceito de violência: Distinções necessárias. In L. C. A. Williams, J. M. D. Maia, \& K.S.A. Rios (Orgs.), Aspectos psicológicos da violência: Pesquisa e intervenção cognitivo-comportamental (pp. 41-66). Santo André, Brasil: ESETec.

Stelko-Pereira, A. C., \& Williams, L. C. A. (2016). Evaluation of a Brazilian school violence prevention program (Violência Nota Zero). Pensamiento Psicológico, 14(1), 63-76. DOI:10.11144/Javerianacali.PPSI141.ebsv

Stelko-Pereira, A. C., Williams, L. C. A., \& Freitas, L. C. (2010). Validade e consistência interna do Questionário de Investigação de Prevalência de Violência Escolar Versão estudantes. Avaliação Psicológica, 9(3), 403-411. http://pepsic.bvsalud.org/ scielo.php? script $=$ sci arttext\&pid $=S 1677$ 04712010000300007\&lng=pt\&nrm=iso

Turner, I., Reynolds, K. J., Lee, E., Subasic, E., \& Bromhead, D. (2014). Well-being, school climate, and the social identity process: A latent growth model study of bullying perpetration and peer victimization. School Psychology Quarterly, 29(3), 320-335. DOI:10.1037/spq0000074

Valle, J. E., Stelko-Pereira, A. C., Sá, L. G. C., \& Williams, L. C. A. (2015). Bullying, vitimização por funcionários e depressão: Relações com o engajamento emocional escolar. Psicologia Escolar e Educacional, 19(3), 463-473. DOI:10.1590/21753539/2015/0193872

Wolke, D., \& Lereya, S. T. (2015). Long-term effects of bullying. Archives of Disease in Childhood, 100(9), 879-885. DOI:10.1136/ archdischild-2014-306667

Yen, C., Lin, I., Liu, T., Hu, H., \& Cheng, C. (2014). Mediating effects of depression and anxiety on the relationship between bullying involvement and pain problems among adolescents in Taiwan. Comprehensive
Psychiatry, 55(6), 1415-1421. DOI:10.1016/j. comppsych.2014.05.006

\section{FinANCIAMENTO}

Agradecemos ao Conselho Nacional de Desenvolvimento Científico e Tecnológico Brasileiro pelo apoio financeiro (processo 408695/2013-7). 


\section{VALIDITY EVIDENCES FOR THE Victimization among Students SCALE (VSS)}

\section{AbstraCt}

This study evaluated the Brazilian instrument Victimization among Students Scale's (VSS) in respect to the internal structure, internal consistence, and its relationship with another variable (stress). A database involving 1.484 students, from grades $6-9$, in public schools of six cities from four Brazilian States was employed. Results indicated that the Scale comprehends two factors: face-toface victimization and virtual victimization, which in conjunction explain about $50 \%$ of the variance, with an internal consistency index of 0,84 and 0,77. Additionally, the VSS was applied twice in a period of 30 days $(\mathrm{N}=100)$ in the city of Fortaleza, with a total reliability score of 0,64. Participants from Fortaleza also answered the Child Stress Scale (CSS), and positive significantly correlations above 0,65 were found between VSS and CSS. Thus, EVA is a promising alternative to evaluate the phenomenon.

KEYworDS: Bullying; School harassment; Psychometric
EVIDENCIAS DE VALIDEZ PARA LA ESCALA DE VICTIMIZACIÓN ENTRE Alumnos (EVA)

\section{RESUMEN}

Este estudio evaluó la Escala de Victimización entre Alumnos (EVA), en cuanto a la validez basada en la estructura interna, consistencia interna, y en la relación con otra variable (estrés). Para ello, se analizaron respuestas al EVA de 1.484 alumnos de escuelas públicas de $6 .^{\circ}$ a $90^{\circ}$ grado de la Educación Básica provenientes de seis ciudades de cuatro estados de Brasil. Se observó que la escala está formada por dos factores: victimización presencial y victimización virtual, los cuales conjuntamente explican cerca del $50 \%$ de la varianza, obteniendo un índice de consistencia interna de 0,84 y 0,77 para los factores. Adicionalmente, en la ciudad de Fortaleza $(\mathrm{N}=100)$ se aplicó a EVA dos veces en un intervalo de 30 días, obteniéndose un índice de precisión para el puntaje global de 0,64, y se aplicó la Escala de Estrés Infantil (EEI), habiendo percibido correlaciones positivas significativas superiores a 0,65 entre los escores de la EVA y de la EEI. Así, EVA es una alternativa prometedora.

Palabras Clave: Bullying; Intimidación escolar; Psicometría

\footnotetext{
${ }^{1}$ Departamento de Teorias e Fundamentos da Educação, Universidade Federal do Paraná - UFPR, Brasil. ORCID: 0000-00028089-132X

${ }^{\text {II }}$ Departamento de Psicologia, Universidade Federal de São Carlos - UFSCar, Brasil. ORCID: 0000-0003-3425-6656

III Programa de Pós-Graduação Stricto Sensu em Psicologia, Universidade São Francisco - USF, Brasil. ORCID: 0000-00023921-8547

Iv Programa de Pós-Graduação Stricto Sensu em Psicologia, Universidade São Francisco - USF, Brasil. ORCID: 0000-00030107-2033
} 